\title{
Image Enhancement using Vector Valued Image Processing
}

\author{
Bhagyashree M. Kamble \\ CSE.Department, Deogiri Institute of \\ Engineering and Management Studies, \\ Aurangabad, $\mathrm{MH}$, India.
}

\author{
Pankaj H.Durole \\ CSE.Department , Deogiri Institute of \\ Engineering and Management Studies, \\ Aurangabad, MH, India
}

\begin{abstract}
The images which consist of RGB color images is known as vector valued images. This images is the multimodal images which shows strong inter-channel correlation among each other. We treat this images by the new notion by introducing the spatial gradient channels. We can obtained the vector valued through minimizing the cost functional that penalizes the large angles. After this we introduce the Gateaux derivative that leads to diffusion gradient decent scheme. The cost functional gives the several examples of denoising and demosaicking. These shows that demosaicking will gives visually perfect image for the low noise by parallel level set preceded by denoising. We get the result that after introducing all the idea to the image we will yield the better result than any other approaches.
\end{abstract}

\section{Keywords}

vector valued images, denoising, demosaicking, diffusion, parallel level sets.

\section{INTRODUCTION}

Many imaging application are there which shows more than one piece of information at single point in an image. Common example of such image is RGB color images, which shows different color i.e. Red, Blue, Green are encoded in some amount at any point. We can think of such images on one hand as three scalar valued image or as single vector valued images. Another example is the scanners which are used in the medical field to take the image of the spatial part of the body, for example (CT) computed tomography scanner which measure the absorption of X-rays by the body or a magnetic resonance (MR) scanner which measures the response of water molecules against the magnetic field. Other than these scanner a positron emission tomography (PET) or a single photon emission computed tomography (SPECT) scanner can obtain functional properties like blood flow, metabolic activity. Advance scanner used in these modern era is the combination of different scanner such SPECT/CT or PET/MR devices from which a vector valued image is obtain where different channel of vector corresponds to different properties of tissues. These properties are interdependent. This dependency is what that we are going to explore [1].

Many image processing tools that are designed for scalar valued images but they are applied to vector valued images they process these images independently channel by channel, this processing fails to explore the information expressed in correlation between channels. The prominent example of using information between channels is color total variation, which further leads to the extension of scalar valued to nonlinear diffusion where there is the dependency of diffusivity on all the channels. Various approach is extended for the vector valued images and variational method are combined using the concept of Polyakov action which will yield Nambu functional. By considering the degree of parallelism between the level sets of each channel we propose new approach which emphasis geometric correlation between channels.In our endeavor we assume that the components are not interdependent, therefore when we attempt for this we get parallel level sets by aligning the gradient between the channels. Hence we will get the similar structure images. We will see the enhancement of the color images using denoising and demosaicking in [2] [3].

One of the prompt example is (CTV) color total variation that uses the information between the channels. This scalar valued version extension leads to a non-linear diffusion scheme where the diffusivity depends on all the channels. such approaches extends several variational methods in image processing of vector valued images which are combined using the concept of Polyakov action which yields the so called Nambu functional

The organization of the paper is as follow. First we will discuss the variational approaches that will be used in image processing, which discuss about the diffusion equation which will occur automatically when gradient based cost functional is introduced. Then we will introduce different approach to handle the parallel level sets, gateaux derivative is introduced and finally several examples of denoising and demosaicking is shown in comparison with the different approaches

\section{RELATED WORK}

The flow of the paper is as shown in the block diagram Figure 1.

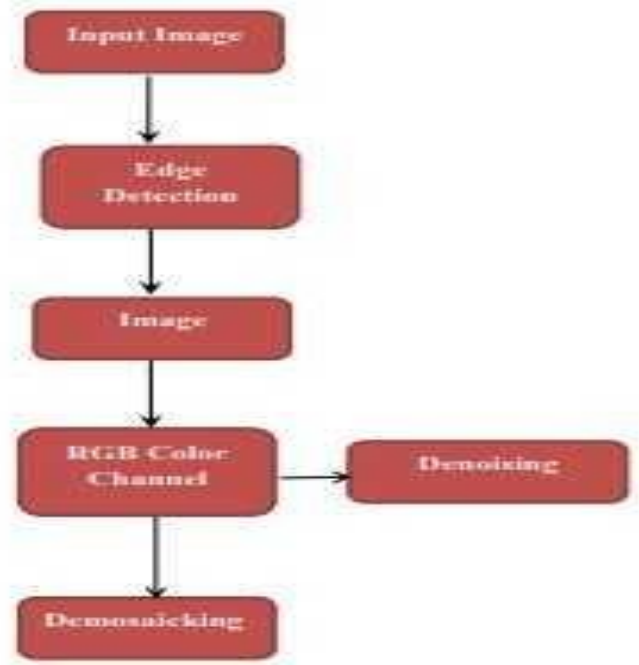

Figure 1 Block diagram 


\subsection{Demosaicking}

Demosaicking is the concept, to reconstruct the digital image when we apply some cost functional properties. It is an algorithm used to reconstruct the digital images to full color image from the incomplete color samples. This concept is called as [CFA] color filter array interpolation or color reconstruction. Bayer filter is the most commonly used CFA which consist of square grids of photosensors. In this method each channel is treated separately in single channel interpolation without utilizing any Inter channel correlation. Different standard techniques used for image interpolation is bilinear / bicubic interpolation and spline interpolation. When we use linear interpolation technique, the frequencydomain interpretation is convenient because the corresponding low pass filter suppress the aliasing by eliminating overlapped high- frequency components. Question here is that how to improve the quality of demosaicking images by effectively exploring their inter channel dependency.

The result of the related work as see Figure 3. The results of image processing using denoising and demosaicking can be seen through the link given in [5]. Sequential interpolation appears to be the most popular techniques in demosaicking literature. The key motivation behind sequential approach is that the luminance (Green) channel is less aliased than the other two. Therefore, having a full resolution luminance channel facilitates the recovery of chrominance (red/blue). The basic steps that demosaicking is as follow see [6].

Reconstruction: In 2D images with three color channel the reconstruction is less trivial. Edges becomes curves rather than points, in many cases one needs to interpolate

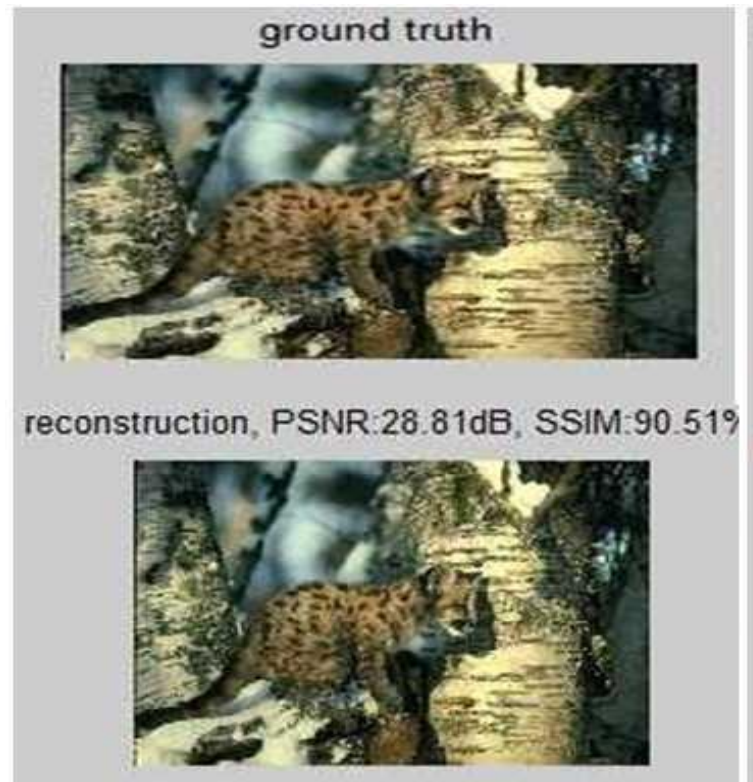

missing points along the edges. Based on simple color image formation model, three channel go through a sudden change across the edges. The gradient $G$ thus can be used as an edge indicator. The directional derivative of the neighbor pixel is denoted by $\mathrm{D}$ at center, $\mathrm{D}+$ for forward and D- for backward as see Figure 2 below.

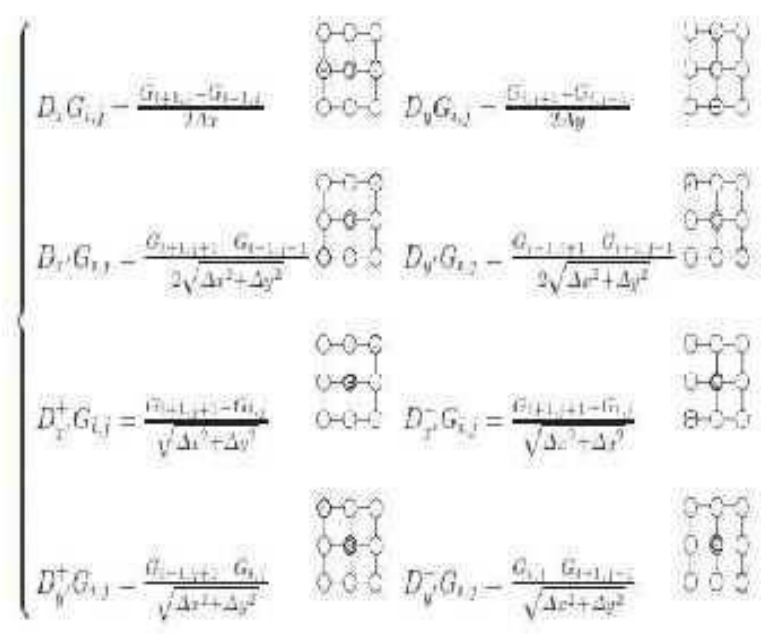

Figure 2 Directional derivative of neighboring pixels.

Enhancement: This section describes in briefly one of the nonlinear filter that we apply as a second step for the enhancement of the color image. Image enhancement procedure is mainly based on an anisotropic flow via inverse second directional derivative in the edge direction and geometric heat equation as a diffusion along the edges. The proposed image enhancement is given in the [4].
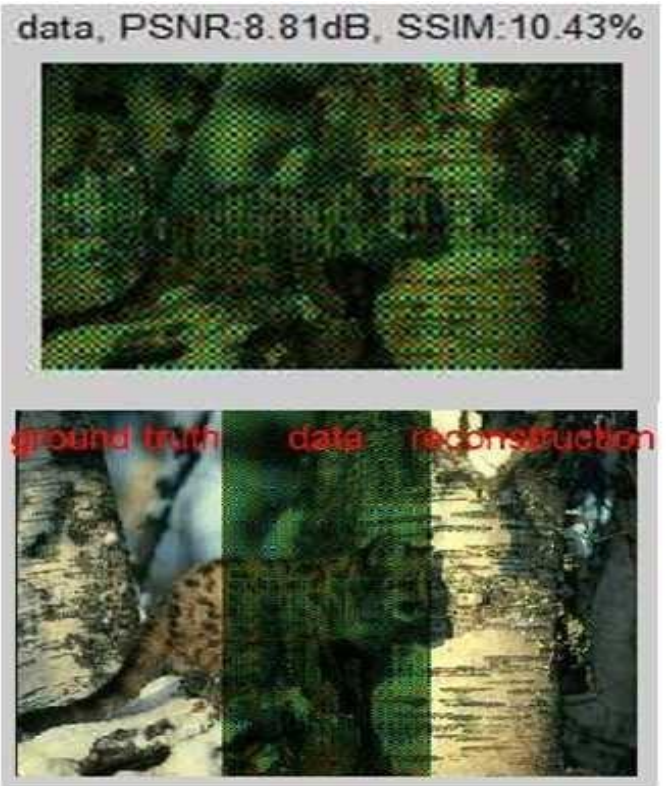

Figure 3. Result of demosaicking. 


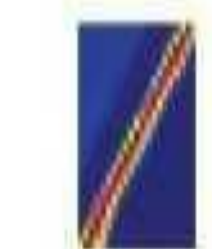

Loal Filier Weightsipinal i)

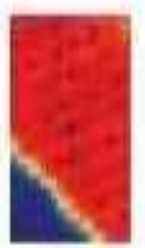

Loul Filter-Wetghisipoul 2

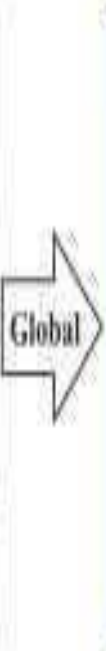

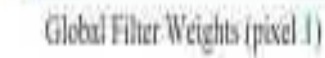

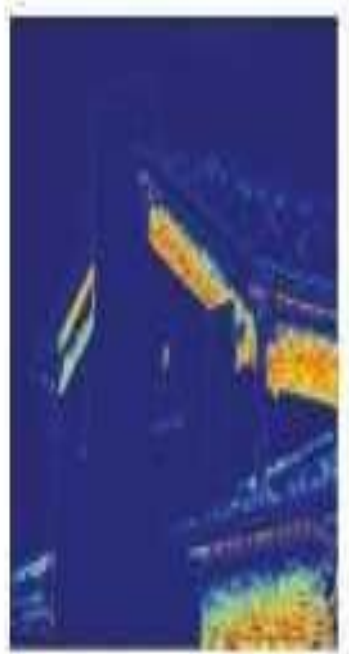

(Glotal Filter Wouthe (pixel ?)

Figure 4. Global filter for denoising
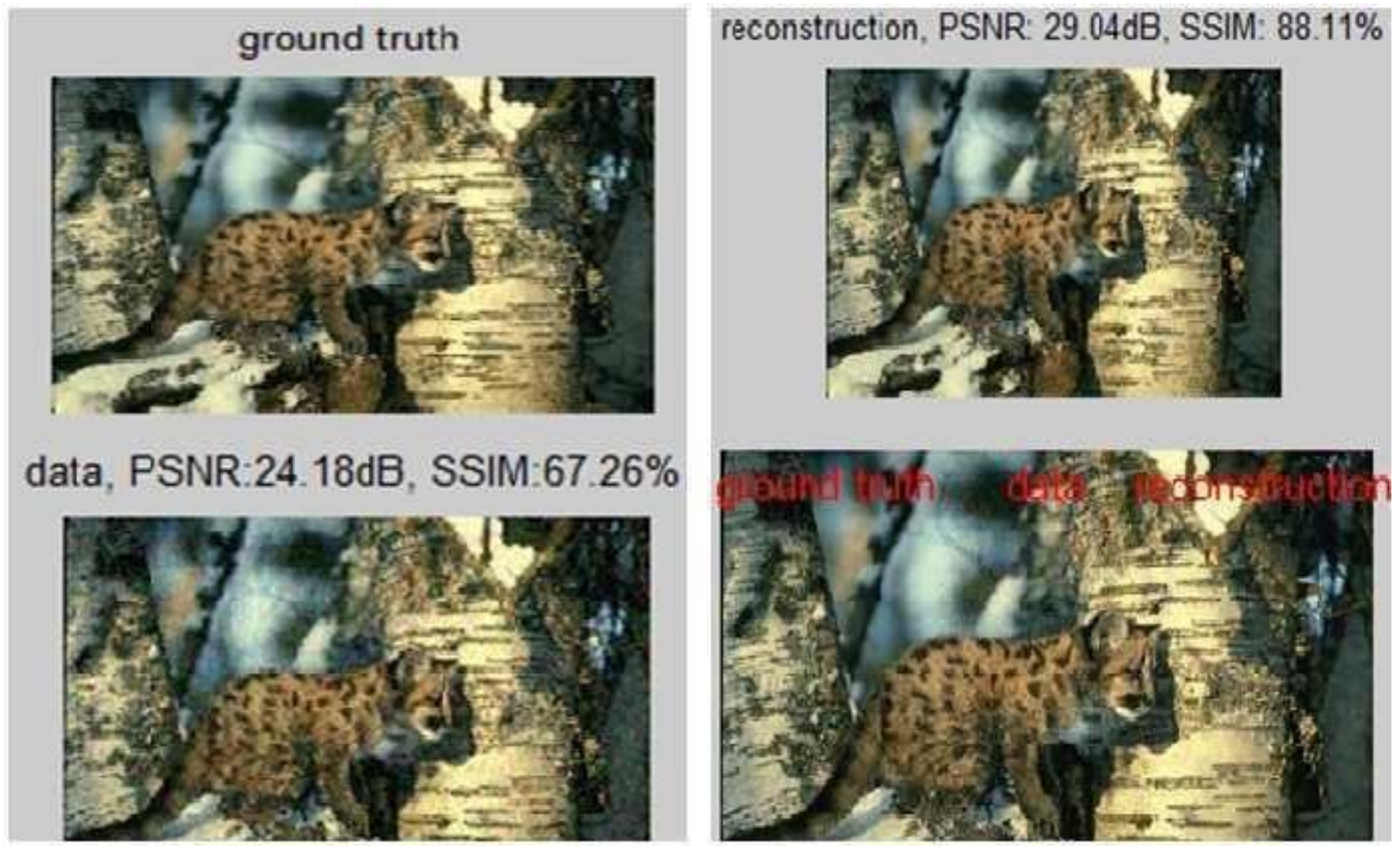

Figure 5 Result image of Denoising

\subsection{Denoising}

The basic concept of denoising is to reduce the noise from the images, this reduction of noise is done through image blurring. We can use this concept of blurring locally, take for example Gaussian smoothing model or by calculus of variation image into account as shown in the Figure 4.

With this global filter in hand the patch based processing concept is no longer restrictive, and we can show that performance of the existing patch based filter are improvable. The block diagram of glide is as shown in the figure and the al estimate of the image to be reconstructed. The result of the denoising image of the related work is as see Figure 5.

By using this method we can show that it is used to denoise the color images. We take the images from the Berkeley Segmentation Database. For denoising of the image we compare our result with the different methods such as Nambu functional, Color total variation and nonlocal mean algorithms. Here we introduced different Gaussian noise in the images with the different standard deviation such as 5,10,15,20 and 35 as shown in the [1]. And the difference between this images and the denoising image is illustrated there in [1].Segmentation 
The image can be divided into meaningful structures using segmentation which often consists of essential step in image analysis, such as object representation, visualization and many other image processing tasks algorithms. A variety of segmentation methods is declared in the past decades, some categorization is.

Threshold based segmentation: It includes Histogram threshold and slicing techniques used to segment the
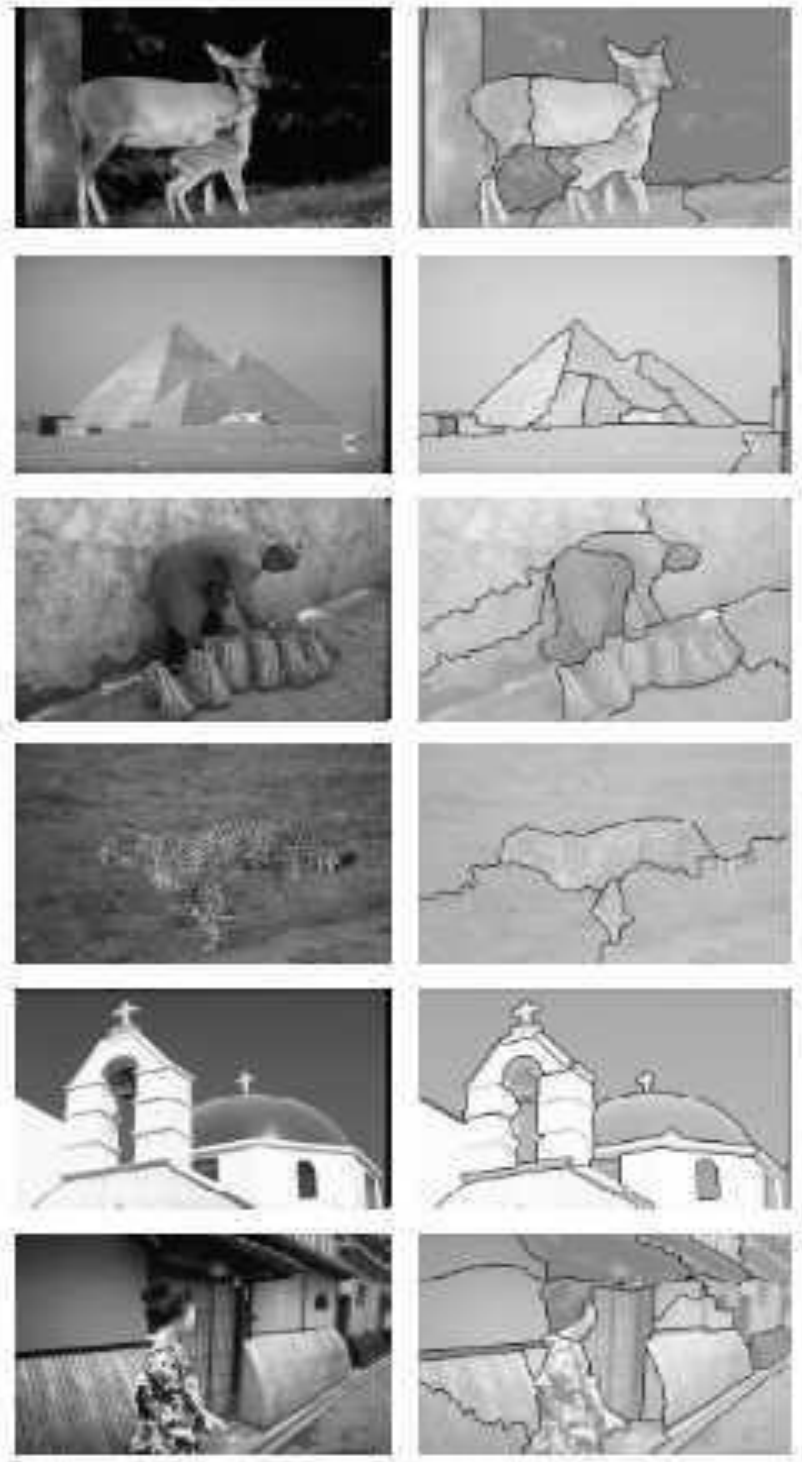

image. They can be applied directly to an image, but can also be combined with pre-processing and post processing techniques.

Edge based segmentation: It include the technique, detection of edges in an image are assumed to represent the object boundaries and used to identify these objects.
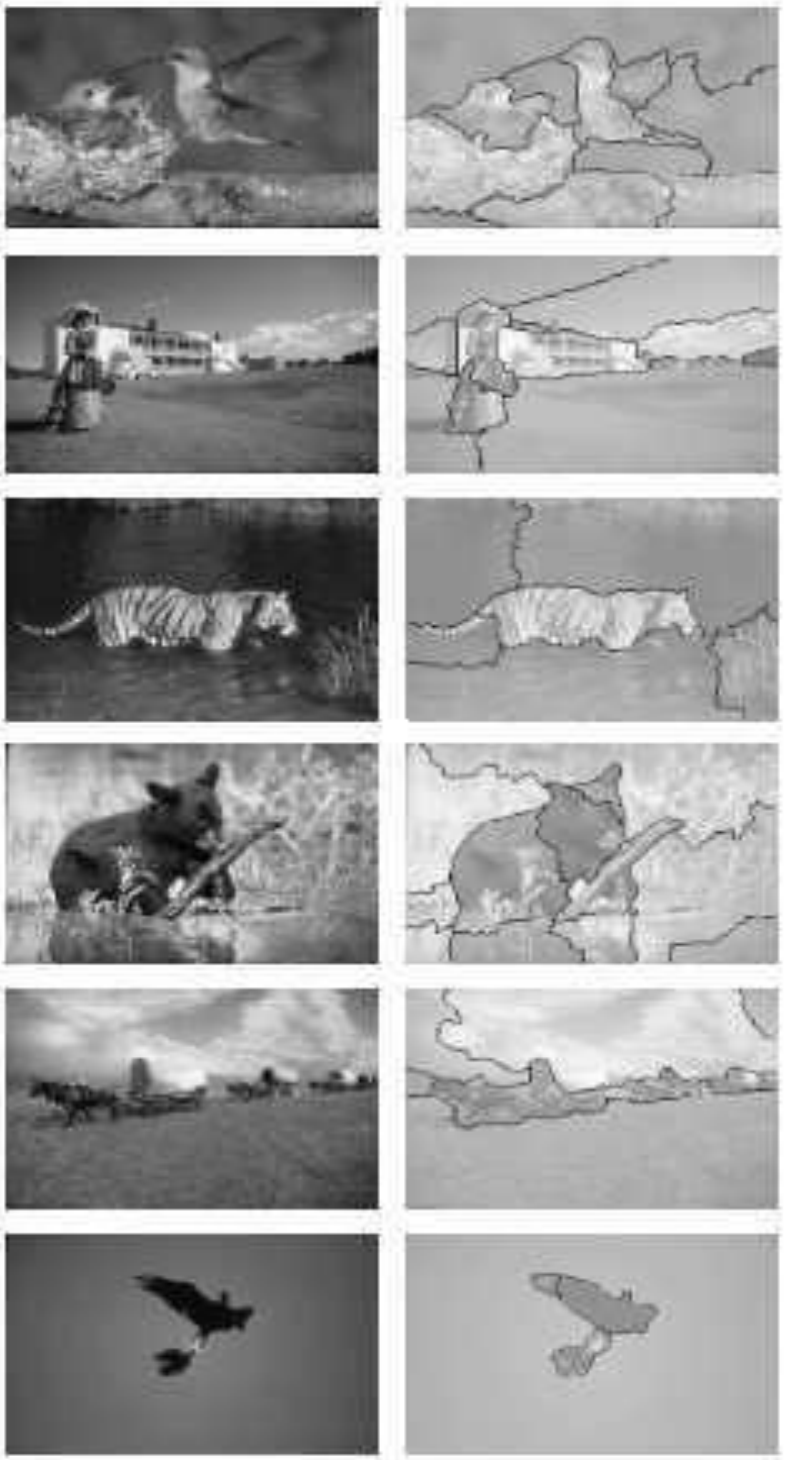

Figure 6 Berkeley Segmentation Database

Region based segmentation: It include an edge based technique which attempt to find the object boundaries and locate them by filling it, this techniqueTake the opposite approach for example, starting in the middle of an object and then growing outwards until it reaches the boundaries.

\section{Clustering techniques}

It is sometimes used as a synonym for segmentation techniques, we use this techniques to denote the primary use of high dimensional measurement patterns. Here this method used in data analysis is attempt to group together Since segmentation of natural images is important as it is often used as the first stage of image analysis algorithms for various tasks such as database organization and retrieval, classification detection and recognition of different patterns that are similar in some sense. The goal of our attempt is quite similar to that when we segment an image, and indeed some of this techniques of clustering can readily be applied for image segmentation. The segmentation of some images is as see Figure 6.

\section{Matching techniques:}

It is the technique in which we know what object we wish to identify in an image approximately looks like, we can thus use this technique to locate the object in an image. This attempt to segmentation is called matching objects in images, compression. Shown in figure. Which illustrate the performance of our algorithm on several images from Berkeley segmentation dataset.

Algorithms 
We are going to discuss some commonly used algorithm [1] of segmentation, clustering algorithms, CVK algorithm.

K-means algorithm

This algorithm is designed to cluster a number of objects into

$\mathrm{K}$ cluster or classes, based on the location of an object. Objective of this algorithm is to assign the object to classes so that they lie closer to the average location of their class than to the average location of other classes.

This algorithm can be used to cluster pixels of a digital image with related color if the pixel are considered objects. Rather than the actual position of the pixel,

location is measured in terms of color pixels are located in the color space not in the physical space. In a class the average location of in color space CVK algorithm of pixels can be calculated by

Averaging the colors of the pixels in the class. The distance between a pixel pi and aj can be expressed by in this CVK algorithm the pixel color is compare to the mean color of its subdomain and the mean colors of the subdomain lie opposite the hypersurfaces. Hence a pixel can stay in its subdomain or move to one of $n$ other subdomain. The k-means algorithm allow the pixel to move to other $2 \mathrm{n}-1$ subdomain. Apparently the pixel might be denied the opportunity to move to the right subdomain.

The CVK algorithm segments an image by evolving hypersurface according to the PDE.

$$
\begin{aligned}
& d\left(p_{i}, a_{j}\right)=\frac{1}{m} \sum_{k=0}^{m-1} \lambda_{j, k}\left(p_{i, k}-a_{j, k}\right)^{2}, \\
& \frac{\partial \phi_{i}(\mathbf{x})}{\partial t}=\nabla \phi_{1}(\mathbf{x}) \mid\left(\mu \nabla \cdot\left(\frac{\nabla \phi_{i}(\mathbf{x})}{\left|\nabla \phi_{l}(\mathbf{x})\right|}\right)-(1-\mu) \operatorname{sign}\left(\phi_{i}(\mathbf{y})\right)\right) \\
& \text { where } \mu \in[0,1] \text { and }
\end{aligned}
$$

$$
\begin{aligned}
& \mathbf{y}=\underset{z \in \Omega}{\arg \min }\left\{\frac{1}{m} \sum_{j=0}^{m-1} \lambda_{i, j}\left(u_{j}(\mathbf{z})-c_{i, j}(\mathbf{z})\right)^{2}\right\} \\
& d\left(p_{i}, a_{j}\right)=\frac{1}{m} \sum_{k=0}^{m-1} \lambda_{j, k}\left(p_{i, k}-a_{j, k}\right)^{2},
\end{aligned}
$$

\section{PROBLEM DEFINITION AND SCOPE}

We have to enhanced the images of vector valued using the technique of denoising and demosaicking. Early CMYK color model was used to enhancing the images but the results was not convincing enough and were not paramount. Thus to improve the quality of the images RGB color model were used and it give the convincing result. RGB model consist of wide range of colors other than CMKY which produces colors that are more vivid and vibrant. CMKY model produced the color beyond the range of RGB color model and are typically dull and dark. In medical field different scanner measure different properties for the spatial point of the body. For example CT (computed tomography) measures the absorption of Xrays.

\section{CONCLUSION}

The framework is based on RGB color model which can be used for image enhancement of vector valued images which overcomes the drawbacks of the CMYK color model. The inter channel correlation is been exploited here which is inherent in many vector valued images such as RGB color images. Examples shown in this paper indicate that exploiting this correlation leads to better, sharper reconstruction of the noisy image. The concept of denoising and demosaicking can be easily extendible to other application where more complicated operator is involved. We can include for instance the simultaneous reconstruction of noisy image by using different techniques of image processing. The related work of the paper can be used in the future for research purpose so that we can yield better images.

\section{REFERENCES}

[1] Vector-Valued Image Processing by Parallel Level Sets Matthias Joachim Ehrhardt, Simon R. Arridge.

[2] B. K. Gunturk, Y. Altunbasak, and R. M. Mersereau, "Color plane interpolation using alternating projections," IEEE Trans. Image Process., vol. 11,no. 9, pp. 997_1013, Sep. 2002.

[3] B. K. Gunturk, J. Glotzbach, Y. Altunbasak, R. W. Schafer, and R. M. Mersereau, "Demosaicking: Color _lter array interpolation," IEEE Signal Process. Mag., vol. 22, no. 1, pp.44_54, Jan. 2005.

[4] Comparative Study of Demosaicing Algorithms for Bayer and Pseudo- Random Bayer Color Filter Arrays Georgi Zapryanov, Iva Nikolov.

[5] M. J. Ehrhardt and S. Arridge. (2013, Jul.). VectorValued Image Processing by Parallel Level Sets [Online].Available:http://www0.cs.ucl.ac.uk/staff/ehrh ardt/software.html.

[6] Demosaicing: Image Reconstruction from Color CCD Samples Ron Kimmel. 